\title{
Online Traffic Sources and Persuasion Techniques: How to Change Consumers' Behaviors
}

\author{
Dimitris Drossos, Manos Zacharioudakis, George Dionysiou \\ Athens University of Economics \& Business \\ University of the Aegean
}

\begin{abstract}
One of the biggest challenges electronic commerce managers face today is how to encourage website visitors to act. Usability is no longer enough, and managers must step forward into the new world of user experience and persuasive design. This paper examines what drives shoppers to click an add-to-cart button, an important micro conversion for e-retailers, since it is the first commitment that a visitor makes towards becoming a customer. Therefore, the purpose of this paper is to examine how two persuasion techniques and three different types of visitors promote website conversions. We conducted an online field experiment and data were collected from more than one thousand users who visited the retailer's website. The results of this experiment reveal that both social proof and scarcity persuasion techniques affect users' behaviors and increase micro-conversion rates. Moreover, visitors from three different traffic channels behave diversely while browsing the retailer's website and interacting with these persuasive techniques. Theoretical and practical implications of these findings are discussed.
\end{abstract}

Keywords: e-commerce; audience; conversion; persuasion; scarcity; social proof 